\title{
Commentary: Severe fetal acidemia in cases of clinical chorioamnionitis in which the infant later developed cerebral palsy
}

\author{
Yoshio Matsuda
}

Department of Obstetrics and Gynecology, International University of Health and Welfare Hospital, Japan

\section{Article Info}

\section{Article Notes}

Received: 15/04/2016

Accepted: 03/05/2016

\section{Correspondence:}

Dr. Yoshio Matsuda

Department of Obstetrics and Gynecology

International University of Health and Welfare Hospital, 537-3

Iguchi Nasushiobara, Tochigi 329-2763, Japan

Telephone: +81-3-3353-8111

Fax: +81-3-5269-7350

Email: yoshiom2979@gmail.com

(c) 2016 Matsuda Y. This article is distributed under the terms of the Creative Commons Attribution 4.0 International License

\section{Commentary on Previous Publication}

Yoshio Matsuda, Masaki Ogawa, Akihito Nakai, Miki Tagawa, Michitaka Ohwada, Tsuyomu Ikenoue Severe fetal acidemia in cases of clinical chorioamnionitis in which the infant later developed cerebral palsy. BMC Pregnancy and Childbirth. 15:124 DOI: 10.1186/s12884-015-0553-9, 2015

URL: http://www.biomedcentral.com/1471-2393/15/124

\section{Background}

Although birth asphyxia may cause cerebral palsy (CP), it has been demonstrated in controlled population-based studies that birth asphyxia does not account for most cases of $\mathrm{CP}^{1}$. It has also been reported that there is a close relationship between $\mathrm{CP}$ and clinically apparent chorioamnionitis (CAM) ${ }^{2,3}$, and $\mathrm{CP}$ has been shown to be induced by the direct effects of cytokines on the brain, even under conditions of mild hypoxic stress ${ }^{3}$. However, the relationships between infection/inflammation and the onset of CP remain unclear. Severe/pathological fetal acidemia is defined as an umbilical arterial $\mathrm{pH}(\mathrm{UApH})$ of less than 7.0 and base deficit $\geqq$ $12 \mathrm{mmol} / \mathrm{l}^{4}$. This condition is an objective measurement of the level of intrapartum hypoxia-ischemia and correlates with the occurrence of hypoxic ischemic encephalopathy ${ }^{4}$.

The Japan obstetric compensation system for $\mathrm{CP}$ was established by the Japan Council for Quality Health Care (JCQHC) to compensate for $\mathrm{CP}$ resulting from intrapartum events and improve perinatal care $^{5,6}$.

The objective of this study was to determine the UApH in CAM cases in which the infant later develop severe CP.

\section{Methods}

This study was approved by the Ethics Committee of the International University of Health and Welfare Hospital, Tochigi, Japan. A review was conducted between January l, 2009 and April 30, 2014 among 324 infants with CP diagnosed to be caused by antenatal and/or intrapartum conditions as determined by the JCQHC. At the diagnosis of cerebral palsy, the mean infant's age was $1.8 \pm 0.4(0.5-2.5)$ years in group 1 , and $1.6 \pm 0.5(0.5-2.7)$ years in group 2, respectively.

CAM was defined as a maternal temperature of $\geqq 38{ }^{\circ} \mathrm{C}$ and at least one of the following four criteria: maternal tachycardia of $\geqq$ $100 \mathrm{bpm}$, uterine tenderness, white blood cell count of $\geqq 15,000$ / $\mathrm{mm} 3$ or foul smelling vaginal discharge ${ }^{7}$.

The FHR pattern observed two hours before delivery was defined as abnormal if one of the following patterns was detected: recurrent late decelerations, minimal or absent variability lasting for 40$60 \mathrm{~min}$, severe variable decelerations, prolonged decelerations, 
tachycardia or bradycardia ${ }^{8,9}$. Tachycardia was defined as a baseline FHR of over $160 \mathrm{bpm}$ and bradycardia was defined as a baseline FHR of less than $100 \mathrm{bpm}(>3 \mathrm{~min})^{9}$.

The causative factors for $\mathrm{CP}$ in 324 cases were classified according to the report by MacLennan (International Consensus Criteria) with modifications ${ }^{10-12}$. After exclusion of other causes, the remaining 86 cases were divided into two groups: cases with (Group I, n=19) and cases without (Group II, $n=67$ ) clinical CAM, in order to clarify the characteristics of the cases of clinical CAM in which the infant later developed CP.

Severe/pathological fetal acidemia was defined as UApH of less than 7.0 and base deficit $\geqq 12 \mathrm{mmol} / \mathrm{l}^{4}$. The distribution of UApH was graded as follows: less than 7.0 (severe/pathological acidemia) and more than 7.0

The statistical analysis was conducted using the chi-square test, Fisher's exact probability test or MannWhitney test, as appropriate. P values of less than 0.05 were considered to be significant. The variables were compared in both univariate and multivariate analyses. Logistic regression models were used to assess confounding effects. Our selection of the risk factors for inclusion in the regression model was based on the clinically important factors as well as the results of a univariate analysis.

\section{Results}

By comparing of the clinical characteristics of the two groups, the frequency of severe acidemia (Group I, 26.3\% and Group II, 74.6\%) and fetal tachycardia (Group I, 57.9\% and Group II, 14.9\%) was significantly different between the two groups. There were no significant differences between the two groups in terms of the other factors (Table 1).

For factors considered to be possible factors_such as $\mathrm{pH}$ less than 7.0 (severe acidemia), base deficit $\geqq 12 \mathrm{mmol} / \mathrm{l}$, prolonged deceleration, severe variable deceleration, and tachycardia, a further analysis was performed using a logistic regression analysis. The results showed that the frequency of severe acidemia was significantly lower in Group I (OR 0.12, 95\% CI 0.03 - 0.53) than in Group II, while the frequency of tachycardia was greater in Group I (OR 7.61, 95\% CI 1.82 - 31.7) than in Group II, after adjusting for confounding effects.

\section{Discussion}

Several mechanisms have been proposed to explain the association between clinical CAM and CP, including an inflammatory cytokine response, placental inflammation leading to impaired placental perfusion and gas exchange and intrauterine infection with bacteremia resulting in the direct involvement of the brain or fever associated with the infection itself exacerbating the primary insult to the brain $^{12}$. Whether the presence of infection/inflammation adds an additional risk for $\mathrm{CP}$ in term infants delivered in the presence of fetal acidosis has not been thoroughly studied. A single severe exposure, such as uterine rupture or massive placental abruption defined as the separation of the placenta from the uterine lining, may be sufficient to cause CP; however, much more often, it is not a single cause, but rather multiple concurrent risk factors, that precede the onset of $\mathrm{CP}$.

In the present study, the frequency of severe acidemia was significantly lower among the cases of clinical CAM with abnormal FHR patterns such as recurrent late decelerations, minimal or absent variability, severe variable decelerations, prolonged decelerations, tachycardia or bradycardia than in those without clinical CAM. This finding indicates that brain injury may occur following a low level of hypoxic stress in the presence of clinical CAM. Garnier et al. $^{13}$ showed that intrauterine exposure to infection severely altered fetal cardiovascular function, resulting in dysregulation of cerebral blood flow and subsequent hypoxic-ischemic brain injury. In chronically instrumented

\begin{tabular}{|c|c|c|c|}
\hline & Group I (with CAM) & Group II (without CAM) & \\
\hline & $\mathrm{n}=19$ & $\mathrm{n}=67$ & $p$ \\
\hline Gestational age at delivery & $38.9 \pm 1.5$ & $39.0 \pm 1.5$ & 0.801 \\
\hline Birth weight (g) & $2947.3 \pm 440.9$ & $2910.6 \pm 517.2$ & 0.759 \\
\hline \multirow{2}{*}{$\mathrm{pH}($ mean $\pm \mathrm{SD}$, range $)$} & $7.07 \pm 0.14$ & $6.87 \pm 0.18$ & \multirow{2}{*}{$<0.0001$} \\
\hline & $(6.82-7.35)$ & $(6.55-7.24)$ & \\
\hline $\mathrm{pH}$ less than 7.0 (severe acidemia) & $5(26.3 \%)$ & $50(74.6 \%)$ & $<0.0001$ \\
\hline Base deficit $\geqq 12 \mathrm{mmol} / \mathrm{l}$ & $11(11 / 17,64.7 \%)$ & $57(57 / 62,91.9 \%)$ & 0.018 \\
\hline Fetal growth restriction & $4(21.1 \%)$ & $14(20.9 \%)$ & 0.76 \\
\hline Instrumental vaginal delivery & $5(26.3 \%)$ & $30(44.8 \%)$ & 0.236 \\
\hline Cesarean section & $6(31.6 \%)$ & $18(26.9 \%)$ & 0.89 \\
\hline Prolonged deceleration & $12(63.2 \%)$ & $58(86.6 \%)$ & 0.048 \\
\hline Minimal or absent variability & $4(21.1 \%)$ & $30(44.8 \%)$ & 0.104 \\
\hline Recurrent late deceleration & $7(36.8 \%)$ & $29(43.3 \%)$ & 0.818 \\
\hline Severe variable deceleration & $2(10.5 \%)$ & $23(34.3 \%)$ & 0.092 \\
\hline Tachycardia & $11(57.9 \%)$ & $10(14.9 \%)$ & $<0.0001$ \\
\hline
\end{tabular}

Table 1: Comparison of the clinical characteristics of the two groups; CAM: Chorioamnionitis 
fetal sheep, intravenous injection of lipopolysaccharide decreased placental blood flow, whereas blood flow to the peripheral organ increased. The decrease in placental blood flow was accompanied by sustained hypotension, hypoxemia, and mixed acidosis causing dysregulation of cerebral blood flow. Because the simultaneous change of FHR pattern was not examined in detail, further study is needed to show the relationship between the change of FHR pattern and the degree of infection.

In this study, we were unable to clarify characteristic FHR patterns associated with clinical CAM in which the infant later developed $\mathrm{CP}$, as discussed by other researchers ${ }^{14}$. For example, it has been postulated that increased cytokines in such cases induce ischemia of the umbilical or uterine vessels, subsequently resulting in variable or late decelerations, in which the fetus may become hypoxic and acidotic. In isolation, tachycardia is poorly predictive of fetal hypoxemia or acidemia, unless accompanied by minimal or absent FHR variability, recurrent late/variable decelerations or both ${ }^{11}$. On the other hand, Sameshima et al. ${ }^{15}$ reported that the development of $\mathrm{CP}$ in pregnancies with intrauterine bacterial infection is associated with tachycardia, but not FHR deceleration patterns or acidemia, suggesting the presence of a different pathophysiology in these cases from that observed in the setting of acute hypoxia-ischemia.

Our data are not derived from a nationwide populationbased cohort, as essential information was masked for personal protection. Nevertheless, the peer review system in Japan is the best clinically available method. It may be argued that our results are influenced by bias due to the small sample size; however, we consider this to be unlikely given the low-frequency nature of the cohort.

Further studies are needed to confirm the relationship between an abnormal intrapartum FHR pattern and the presence of clinical CAM by collecting data in such cases prospectively, including those in which the infant later develops CP.

\section{Conclusions}

The frequency of severe acidemia was lower in the cases of clinical CAM in which the infant later developed severe cerebral palsy than in the cases without clinical CAM. The relation of fetal tachycardia to CP with clinical CAM, but not to acidemia, should be reevaluated in such cases.

\section{References}

1. Nelson KB. What proportion of cerebral palsy is related to birth asphyxia? J Pediatr. 1988; 112: 572.

2. Wu YW, Colford Jr JM. Chorioamnionitis as risk factor for cerebral palsy: a meta-analysis. JAMA. 2000; 284: 1417-1424.

3. Kendall G, Peebles D. Acute fetal hypoxia: the modulating effect of infection. Early Hum Dev. 2005; 81: 27-34.

4. Hankins GDV, Speer M. Defining the Pathogenesis and Pathophysiology of Neonatal Encephalopathy and Cerebral Palsy Obstet Gynecol. 2003; 102: 628-636.

5. Matsuda Y, Umezaki H, Ogawa M, Ohwada M, Satoh S, Nakai A. Umbilical arterial pH in patients with cerebral palsy. Ear Dev Hum. 2014; 90: 131-135.

6. The Japan council for Quality Health care. Guide to The Japan obstetric compensation system for Cerebra1 Palsy. http://www.sanka-hpJcqhc. orjp/pdf/bira_english_color201109.pdf.

7. Lencki SG, Maciulla MB, Eglinton GS. Maternal and umbilical cord serum interleukin levels in preterm labor with clinical chorioamnionitis. Am J Obstet Gynecol. 1994; 170: 1345-1351,

8. Matsuda Y, Konno J, Ogawa M, Mitani M, Matsui H. Prediction of fetal acidemia in placental abruption BMC Pregnancy and Childbirth. 2013, 13:156. DOI:10.1186/10.1186/1471-2393-13-156

9. Okai T, Ikeda T, Kawarabayashi T, Kozuma S, Sugawara J, Chisaka H, et al. Intrapartum management guidelines based on fetal heart rate pattern classification Journal of Obstetrics and Gynaecology Research. 2010; 36(5): 925-928.

10. MacLennan A. A template for defining a causal relation between acute intrapartum events and cerebral palsy: international consensus statement. BMJ. 1999; 319: 1054-1059.

11. Kodama Y, Sameshima H, Ikeda T, Ikenoue T. Intrapartum fetal hear rate patterns in infants ( $\geqq 34$ weeks) with poor neurological outcome. Ear Hum Dev. 2009; 85: 235-238.

12.Shalak LF, Perlman JM. Infection markers and early signs of neonatal encephalopathy in the term infant. Ment Retard Dev Disabil Res Rev. 2002; 8: 14-19.

13.Garnier Yves, Coumans ABC, Jensen A, Hasaart THM, Berger R. Infection-Related Perinatal Brain Injury: The Pathogenic Role of Impaired Fetal Cardiovascular Control. J Soc Gynecol Investig. 2003; 10: 450-459.

14.Freeman RK. Problems With Intrapartum Fetal Heart Rate Monitoring Interpretation and Patient Management Obstet Gynecol. 2002; 100: 813-826.

15. Sameshima H, Ikenoue T, Ikeda T, Kamitomo M, Ibara S. Association of nonreassuring fetal heart rate patterns and subsequent cerebral palsy in pregnancies with intrauterine bacterial infection. Am J Perinatol. 2005; 22(4): 181-187. 